\title{
Targeting the indoleamine 2,3-dioxygenase pathway in cancer
}

\author{
Yong Wha Moon ${ }^{1,2}$, Joud Hajjar ${ }^{3}$, Patrick Hwu ${ }^{4}$ and Aung Naing ${ }^{2^{*}}$
}

\begin{abstract}
Tumor cells escape the immune surveillance system of the host through a process called immune tolerance. Immunotherapy targets molecules that serve as checks and balances in the regulation of immune response. Indoleamine-2,3-dioxygenase (IDO) is an intracellular enzyme, which through the process of tryptophan depletion exerts an immunosuppressive effect, facilitating immune escape of tumors. This review summarizes our current knowledge on IDO expression in malignancies, the IDO inhibitors that are currently available and those under clinical development.
\end{abstract}

Keywords: Indoleamine 2,3-dioxygenase, IDO inhibitors, Immune surveillance, Immunomodulatory, Malignancy

\section{Introduction}

Most tumors express potentially immunogenic antigens to which the immune system can respond [1]. In turn, the tumor-bearing host possesses high-avidity $\mathrm{T}$ cells that are specific to these antigens [2]. And yet, in a phenomenon called immune tolerance, tumor cells evolve to escape immune surveillance [3], and the host fails to reject the tumor. A number of molecular mechanisms underlying this induced tolerance have been identified [4]. Immunotherapies targeting such mechanisms have become an important therapeutic strategy for harnessing the immune system's ability to control malignancy, overcoming immune tolerance.

Indoleamine-2,3-dioxygenase (IDO) is an intracellular heme-containing enzyme that initiates the first and ratelimiting step of tryptophan degradation along the kynurenine pathway [5]. In mammalian organisms, tryptophan is an essential amino acid for cell survival; it cannot be synthesized de novo. Initially, the role of IDO was thought to be mainly antimicrobial by reducing the availability of tryptophan in the inflammatory environment [6-8]. IDO was shown to be expressed in normal tissues such as the endothelial cells in the placenta and lung, the epithelial cells in the female genital tract, and the lymphoid tissues in mature dendritic cells (DCs) [9].

\footnotetext{
* Correspondence: anaing@mdanderson.org

${ }^{2}$ Department of Investigational Cancer Therapeutics, The University of Texas MD Anderson Cancer Center, 1515 Holcombe Blvd, Box 455, Houston, TX 77030, USA

Full list of author information is available at the end of the article
}

An innovative discovery by Munn and colleagues showed that IDO has a central role in preventing $\mathrm{T}$ cell-driven rejection of allogeneic fetuses during pregnancy as trophoblast expressing IDO was found to induce maternal tolerance to fetal allograft [10]. This discovery broke ground for further research addressing the immunomodulatory potential of IDO, including a series of studies focused on the role of IDO in the immune escape of tumors, reviewed earlier by Zou [4]. The immunosuppressive roles of IDO have also been investigated for elucidation of therapeutic targets in the management of autoimmune diseases [11] and for induction of graft tolerance after transplantation [12-15].

Several reviews discussing the enzymatic activity of IDO have been published [16-22]. The aims of this article are however, to summarize the most recent data on IDO expression in malignancies and to discuss the current status of clinical testing of IDO inhibitors.

\section{Review}

Mechanisms of IDO pathway involvement in immune tolerance

IDO is an important enzyme in both cancer development and cancer progression. IDO supports inflammation in the tumor microenvironment, development of immune tolerance to tumor antigens in stromal and immune cells, suppression of $\mathrm{T}$ and natural killer cells, generation and activation of $\mathrm{T}$ regulatory cells 
(Tregs) and myeloid-derived suppressor cells, and promotion of tumor angiogenesis [23].

Growing tumors evade the immune system of the host through a complex process of immunoediting in the tumor, where selective pressures result in the outgrowth of tumor clones capable of evading and suppressing active immunity, which often includes expression of tolerance mechanisms. The IDO pathway is one of the endogenous pathways used often by tumor cells to induce tolerance to tumor antigens [21].

As noted above, IDO is expressed by endothelial cells [24], mesenchymal stromal cells, fibroblasts [25], and various myeloid-derived antigen-presenting cells such as DCs and macrophages [26], as well as by tumor cells $[23,27]$. However, some discrepancies in IDO expression profiles in normal and tumor tissues as well as cell types expressing IDO exist. For example, high expression of IDO in tumor draining lymph nodes (TDLN) was associated with poor clinical outcomes $[28,29]$. However, recently it has been reported that TDLNs do not contain more IDO+ cells than normal lymph nodes in a study conducted in 15 patients with breast cancer and 15 patients with melanoma [9]. Similarly, multi-fold increase in expression of IDO was reported with mature DCs but not in immature DCs $[9,30]$. On the contrary, some studies have reported expression of IDO1 by immature DCs or plasmacytoid DCs [28, 29]. Despite these discrepancies, the expression of IDO by antigen-presenting cells can lead directly to suppression of tumor-specific T-cell responses [27] and to activation of Tregs [31]. These effects can be achieved via multiple mechanisms, as demonstrated in Fig. 1.

An important mechanism by which IDO affects T-cell activity is that the consumption of local tryptophan inhibits mechanistic target of rapamycin (serine/threonine kinase) complex 1 (mTORC1) [32] as well as the T-cell receptor regulatory kinase, protein kinase $C$ theta (PKC$\theta)$ [32], both of which are regulatory targets of the master amino acid-sensing kinase, glucokinase (GLK1) [33]. mTORC1 inhibition can trigger a response that includes activating autophagy, leading to anergy in $\mathrm{T}$ cells in the tumor microenvironment [21].

IDO expression can further induce a stress response in cells via activation of general control nondepressible-2 (GCN2). Tryptophan degradation by IDO causes local tryptophan deficiency, leading to the accumulation of uncharged tryptophan transfer ribonucleic acid (tRNA) in cells. GCN2, a stress-response kinase, is stimulated by elevations in uncharged tRNA, and its activation can limit or alter protein translation [34] and prevent T-cell activation [35, 36]. Furthermore, activation of GCN2 promotes de novo Treg differentiation and enhances Treg activity, resulting in profound immunosuppressive microenvironment [36, 37].

An additional mechanism by which IDO expression alters the tumor microenvironment and favors immune

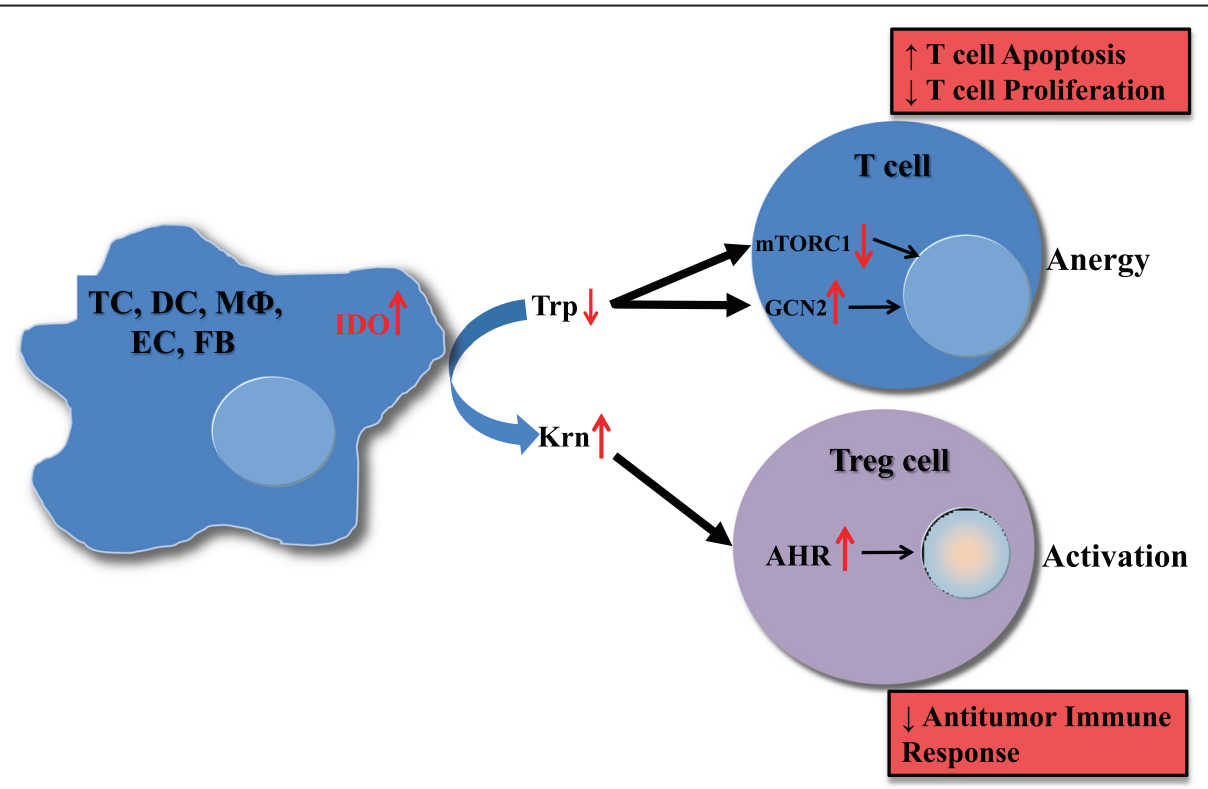

Fig. 1 Mechanisms of IDO pathway activity in immune tolerance. The enzyme IDO catalyzes the initial and rate-limiting step in the catabolism of tryptophan along the kynurenine pathway. Accordingly, IDO can provoke tryptophan shortage, which results in mTORC1 inhibition and GCN2 activation, in turn leading to anergy of effector T cells. Tryptophan's degradation leads to production of bioactive kynurenine pathway compounds, which activate the AHR, resulting in promotion of Treg differentiation. TC, tumor cell; DC, dendritic cell; M $\Phi$, macrophage; EC, endothelial cell; FB, fibroblast; IDO, indoleamine-2,3-dioxygenase 1; Trp, tryptophan; Krn, kynurenine; mTORC1, mechanistic target of rapamycin (serine/ threonine kinase) complex 1; GCN2, general control nondepressible-2; AHR, aryl hydrocarbon receptor; Treg, regulatory T cell 
tolerance is through the production of bioactive compounds. One such compound, kynurenine, which is produced by enzymatic activity of IDO on tryptophan, activates transcription of the aryl hydrocarbon receptor (AHR). Through the AHR, kynurenine metabolites promote differentiation of forkhead box P3-positive $\left(\mathrm{FOXP3}^{+}\right)$Tregs [38] and suppression of antitumor immune responses [39]. All three effects of AHR pathway signaling lead to anergy of effector $\mathrm{T}$ cells and activation of Tregs.

Sharma, Munn and colleagues showed that in melanoma mouse models, IDO is expressed by DCs in the tumor-draining lymph nodes (where tumors are exposed to the antigen-presenting cells for the first time), leading to potent suppression of cluster of differentiation $(\mathrm{CD}) 8^{+} \mathrm{T}$-cell responses and to systemic tumor tolerance [40-42]. Those DCs, when harvested from the tumor-draining nodes and adoptively transferred into new hosts, led to suppression of T-cell responses in vitro, as well as antigen-specific T-cell anergy in vivo $[29,43]$. In mouse models, IDOexpressing DCs activated Tregs, which then systemically suppressed antitumor T-cell responses [37]. The increases in Treg activity are achieved by activation of existing natural Tregs and by upregulation of FOXP3 expression in $\mathrm{CD}_{4} \mathrm{~b}^{+} \mathrm{T}$ cells so that they differentiate into new inducible Tregs [37, 42, 44]. Similar studies showed that IDO-expressing plasmacytoid DCs $[45,46]$ and IDO-expressing monocyte-derived (myeloid) DCs [22] induced differentiation of $\mathrm{CD}^{+}$ cells into FOXP3 ${ }^{+}$Treg-like cells.

\section{Role of the IDO pathway in cancers}

Aberrant IDO activity has been associated with a wide variety of non-oncologic human pathological conditions, including autoimmune diseases, infectious diseases, depression, obesity, organ and bone marrow transplantation and atherosclerosis. Direct evidence of IDO's role has been obtained by studying patients, as well as relevant animal models [47-52].

Several lines of in vitro $[53,54]$ and in vivo $[27,29]$ evidence suggest that the IDO pathway plays a key role in regulating immune evasion by tumors. Recent evidence has demonstrated that the functionally active IDO protein is expressed in a wide variety of human hematologic malignancies, such as acute monocytic leukemia, [55] acute lymphocytic leukemia, [55] acute myeloid leukemia [56] and T-cell leukemia/lymphoma [27], and solid tumors, such as breast cancer [27, 57], colorectal cancer [27], endometrial cancer [27], gastric cancer [27], glioblastoma [27], gynecological cancers [58], head and neck cancers [27], non-small cell lung cancer (NSCLC) [27], small cell lung cancer [27], melanoma [27], mesothelioma [27], and pancreatic cancer [27].
In contrast, most normal cells of the stroma were found to be negative for IDO expression [27]. Several studies have attempted to link IDO activity with its proposed mechanism of action, demonstrating that IDO expression/activity is associated with reduced intratumoral $\mathrm{T}$-cell infiltration, disease progression, and decreased shorter overall survival. For example, an increased kynurenine/tryptophan ratio in the blood was associated with a shorter survival time in patients with acute myeloid leukemia [59]. In patients with solid tumors, such as colorectal cancer [60, 61], endometrial cancer [62, 63], small cell lung cancer [64], melanoma [65], and ovarian cancer [66, 67], high IDO expression is correlated with a poor prognosis and shorter overall survival. The shorter survival of patients whose tumors overexpress IDO supports the concept that a treatment strategy of IDO blockade will have antitumor effects. IDO is thus an attractive target for therapeutic intervention.

IDO may also be involved in mechanisms leading to chemoresistance in cancer patients. In a study of gene expression profiling associated with paclitaxel resistance in patients with serous ovarian cancer, IDO was the most prominently expressed gene. This finding was confirmed with real-time reverse transcription-polymerase chain reaction and immunohistochemistry [67].

Furthermore, the regulation of IDO was demonstrated to contribute substantially to the antitumor effects of imatinib in a mouse model of spontaneous gastrointestinal stromal tumor [68]. Imatinib activated $\mathrm{CD}^{+} \mathrm{T}$ cells and induced Treg apoptosis within the tumor by reducing tumor cell expression of IDO [68]. It is speculated that concomitant immunotherapy with an IDO inhibitor may further improve outcomes in gastrointestinal stromal tumor treated with imatinib.

\section{IDO inhibition as a therapeutic strategy}

There has been increasing scientific interest in IDO as a novel therapeutic target for the development of new cancer drugs, based on the in vitro and in vivo evidence for immune tolerance in the setting of IDO expression in tumor cells and the clinical evidence for poor prognosis and chemoresistance in tumors with high IDO expression. Indeed, potential IDO-inhibiting drugs for use in human cancers are now the focus of research and development efforts. Current inhibitors of IDO are listed in Table 1.

Among the IDO inhibitors, 1-methyl-DL-tryptophan (1-MT) has been the most widely studied. There are 2 available stereoisomers of 1-MT, D and L isomers, with potentially different biochemical and antitumor activity [69]. Pioneering work performed by Hou et al revealed cell-type specific variations in the activity of the 1-MT isomers. In in-vitro studies, the $\mathrm{L}$ isomer (L-1MT) is 
Table 1 Reported IDO inhibitors

\begin{tabular}{lll}
\hline Agent & Mechanism & First author, year \\
\hline D-1MT & Tryptophan mimetic; D isoform of MT; Transcriptional suppressor of IDO & Metz 2012 [32] \\
L-1MT & Tryptophan mimetic; L isoform of MT; selective IDO1 inhibitor & Opitz 201 [39] \\
MTH-Trp & Tryptophan mimetic; transcriptional suppressor of IDO & Okamoto 2007 [92] \\
B-carbolines & Tryptophan mimetic; IDO and TDO inhibitor & Eguchi 1984 [93] \\
Naphthoquinone-based & Pharmacophore of natural product annulin B; indole mimetic; IDO inhibitor & Kumar 2008 [94] \\
S-allyl-brassinin & Phytoalexin; indole mimetic & Gaspari 2006 [95] \\
S-benzyl-brassinin & Phytoalexin; indole mimetic & Gaspari 2006 [95] \\
5-Bromo-brassinin & Phytoalexin; indole mimetic & Banerjee 2008 [96] \\
Phenylimidazole-based & Computationally designed synthetic IDO inhibitor & Kumar 2008 [94] \\
4-phenylimidazole & Heme ligand in IDO enzyme & Sono 1989 [97] \\
Exiguamine A & Non-tryptophan analogue & Harry 2006 [98] \\
NSC401366 & Non-indolic IDO inhibitor & Vottero 2006 [99] \\
\hline
\end{tabular}

IDO Indoleamine-2,3-dioxygenase 1, 1MT 1-methyl-DL-tryptophan, MTH-Trp methylthiohydantoin-dl-tryptophan, TDO tryptophan-2,3-dioxygenase

superior in inhibiting the enzymatic activity of IDO (kynurenine production from tryptophan) in cell-free assays, and several cell lines [69]. Nevertheless, D isomer (D-1MT) is equally effective in inhibiting the enzymatic activity of IDO in human monocyte-derived DCs in allogeneic mixed lymphocyte reactions (MLRs) and is significantly superior to L-1MT or DL mixture in inducing T-cell proliferation in allo-MLRs using either human $\mathrm{T}$ cells stimulated by IDO-expressing monocyte-derived DCs or murine $\mathrm{T}$ cells stimulated by IDO-expressing plasmacytoid DCs from tumordraining lymph nodes [69]. However, this effect is not observed in MLRs using IDO-KO DCs, indicating that the D-1MT exerted its effect in allo-MLRs directly by its action on the IDO gene [69]. Hou et al attributed the cell-type specific variations in the activity of the 1-MT isomers to the possible existence of IDO isoforms. In addition, a statistically significant prolongation of survival is observed with D-1MT in combination with cyclophosphamide in orthotopic 4 T1-luc tumors and in combination with paclitaxel in the autochthonous MMTV-Neu breast tumor model, compared to L-1MT [69]. The superiority of one isomer over the other is therefore dependent on the biological context of IDO expression, tumor cells versus host dendritic cells. Based on the above findings, D-1MT has been considered more suitable for further clinical development. Soon after, Metz et al reported the discovery of a novel IDO-related tryptophan-catabolizing enzyme, IDO2 that is coded by a gene, IDO2, upstream of the INDO gene that codes for IDO (dubbed IDO1). Though IDO2 shares structural similarity to IDO1 [70], IDO2 is expressed only in a subset of tissues that express IDO1 but includes DCs [71]. Consistent with the observations made by $\mathrm{Hou}$ et al, Metz et al demonstrated that L$1 \mathrm{MT}$ modestly inhibits IDO1 activity, whereas D-1MT inhibits IDO2. This possibly explains the greater antitumor activity of D-1MT in preclinical models. Despite the superior antitumor activity of D-1MT, the response to treatment may be negated by the existence of functionally inactive polymorphisms in the IDO2 gene that ablate the enzymatic activity in approximately $50 \%$ of white populations and $25 \%$ of individuals of African descent [71]. The genetic status of the patient regarding IDO2 gene may serve as a predictive marker of response.

Contrary to these findings, Lob et al reported that D-1MT may not be an effective therapeutic option based on a study in a subset of human DCs [72]. Following treatment of interferon- $\gamma$-treated mature DCs with IDO1-specific siRNA, IDO1 transcription was silenced; while expression of IDO2 was unaffected. In addition, tryptophan and kynurenine concentrations were restored following treatment with IDO1-specific siRNA. Similar results were observed in tumor samples that were known to constitutively express IDO [73]. Based on these findings, Lob et al concluded that the IDO activity is only due to IDO1; and IDO2 though expressed by human DCs, was functionally inactive. Therefore inhibition of IDO activity in human DCs by D-1MT was considered unlikely, despite its activity in mice [73, 74]. More recently, genetic ablation of IDO1 alone was sufficient to phenocopy IDO-inhibition with either D-1MT or combined D- and L-1MT regarding important biomarkers of immune activation after chemo-radiation therapy in a mouse brain tumor model [75]. Based on this finding therapeutic inhibition of IDO pathway largely directed against IDO1 has been explored [76]. Despite the inability or poor ability to inhibit the enzymatic activity of IDO, D$1 \mathrm{MT}$ exhibits superior antitumor activity. It is speculated that it interferes with IDO function at other levels besides its inhibitory action on IDO2 [32]. An incidental finding 
that D-1MT acts as a potent tryptophan mimetic in mTOR regulation by restoration of mTOR pathway is one such possible mechanism of action [32]. Further, IDO-mediated catabolism of tryptophan also inhibits the immunoregulatory kinases mTOR and PKC- $\theta$, in addition to inducing autophagy. These effects were also relieved specifically by tryptophan and D-1MT [32]. On the contrary, the immunostimulatory effect of L-1MT is limited by activation of the AHR pathway in response to production of N-methyl-kynurenine, a metabolite of L-1MT. These factors help to explain why L-1MT is considered a poor physiological inhibitor of IDO in comparison to D-1MT and why D-1MT has broader clinical uses against cancers that overexpress any tryptophan catabolic enzyme, such as IDO1, IDO2 and tryptophan-2,3-dioxygenase (TDO) [32].

\section{IDO inhibitors under clinical development}

Currently, four IDO inhibitors are under clinical development (Table 2): INCB024360 (developer: Incyte) [76, 77], indoximod (D-1MT; developer: NewLink Genetics) [32], an IDO peptide vaccine (developer: Copenhagen University) [78], and NLG919 (developer: NewLink Genetics, recently licensed to Genentech) [79].

INCB024360 is an orally administered hydroxyamidine small molecule inhibitor of IDO1. The findings of a phase I study of this IDO inhibitor were reported during a poster discussion session at the 2013 American Society of Clinical Oncology (ASCO) annual meeting [80]. This study was an open-label, single-agent doseescalation trial in 52 patients with advanced malignancies (NCT01195311). Using two independent pharmacodynamic assays, IDO1 inhibition was observed in all patients receiving the compound. Treatment with $\geq$ $300 \mathrm{mg}$ INCB024360 twice a day resulted in greater than $90 \%$ inhibition of IDO1 activity, and the drug was well tolerated. The most common grade 1 or 2 adverse events were fatigue and gastrointestinal disturbances, and the most common grade 3 or 4 adverse events were abdominal pain, hypokalemia and fatigue. The maximum tolerated dose was not established, but based on the pharmacokinetic and pharmacodynamic data indicating that prespecified target inhibition was achieved and on the available tablet strengths, the recommended dose for a phase II study was $600 \mathrm{mg}$ twice daily. The compound led to stable disease for greater than 8 weeks in approximately $30 \%$ of these patients with highly refractory disease; half of the patients had colorectal cancer, which has been refractory to most immunotherapy approaches. Together, these results suggest that INCB024360 has the potential to be effective as monotherapy, but, since it was so well tolerated, it may be particularly useful in combination with other cancer agents. Currently, two phase II trials of INCB024360

Table 2 Clinical trials using IDO inhibitors

\begin{tabular}{|c|c|c|c|c|c|}
\hline Drug & Phase & Mono or combo & Cancer & Clinical trial ID & Comments \\
\hline \multirow[t]{8}{*}{ INCB 024360} & । & Mono & All & NCT01195311 & ASCO 2013 \\
\hline & $\mid \mathrm{b} / \|$ & With ipilimumab & Melanoma & NCT01604889 & ASCO 2014/Recruiting \\
\hline & $\|$ & With MELITAC 12.1 & Melanoma & NCT01961115 & Recruiting \\
\hline & $\|$ & Mono & Ovary & NCT01685255 & Recruiting \\
\hline & $\|$ & Mono & MDS & NCT01822691 & Recruiting \\
\hline & $\mid \mathrm{b} / \|$ & With MK3475 & All/NSCLC & NCT02178722 & Recruiting \\
\hline & $\mathrm{lb} / \mathrm{ll}$ & With CDX-1401, poly ICLC & Ovary & NCT02166905 & Recruiting \\
\hline & Pilot & Mono & Ovary (neoaduvant) & NCT02042430 & Recruiting \\
\hline \multirow[t]{9}{*}{ Indoximod (NLG2101) } & 1 & Mono & All & NCT00739609 & ASCO 2012 \\
\hline & $\mathrm{Ib}$ & With docetaxel & All & NCT01191216 & ASCO 2013 \\
\hline & $|\mathrm{b} /| \mathrm{I}$ & With AD.p53 DC vaccine & All/breast & NCT01042535 & ASCO 2013/Recruiting \\
\hline & $\|$ & With docetaxel & Breast (HER2-) & NCT01792050 & Recruiting \\
\hline & $\|$ & Mono & Prostate & NCT01560923 & Recruiting \\
\hline & $\|$ & With sipuleucel-T & Prostate & NCT01560923 & Recruiting \\
\hline & $\mathrm{Ib} / / \mathrm{I}$ & With nab-paclitaxel, gemcitabine & Pancreas & NCT02077881 & Recruiting \\
\hline & $\mid \mathrm{b} / \|$ & With ipilimumab & Melanoma & NCT02073123 & Recruiting \\
\hline & $\mid \mathrm{b} / \|$ & With temozolomide & Brain & NCT02052648 & Recruiting \\
\hline \multirow[t]{2}{*}{ IDO peptide vaccine } & । & With imiquimod, montanide & $\mathrm{NSCLC}$ & NCT01219348 & Iversen 2013 [78] \\
\hline & $\mathrm{Ib}$ & With temozolomide & Melanoma & NCT01543464 & Recruiting \\
\hline NLG919 & । & Mono & All & NCT02048709 & Recruiting \\
\hline
\end{tabular}

DC dendritic cell, MDS myelodysplastic syndrome, NSCLC non-small cell lung cancer 
monotherapy are recruiting patients with ovarian cancer (NCT01685255) or myelodysplastic syndrome (NCT018 22691). Furthermore, INCB024360 monotherapy is being tested in a neoadjuvant pilot study in ovarian cancer (NCT02042430).

Combining INCB024360 and other immunotherapy modalities such as a cancer vaccine or immune checkpoint inhibitor is being actively investigated based on preclinical data showing synergism of IDO inhibitors and other immune checkpoint blockade strategies [81, 82]. In a phase Ib study of INCB024360 in combination with ipilimumab in patients with advanced melanoma (NCT01604889), which was presented at the 2014 ASCO annual meeting [83], tumor response and duration data suggested the potential for enhanced outcomes with combination treatment compared to ipilimumab monotherapy in melanoma patients. Furthermore, a phase Ib/II study of INCB024360 in combination with another immune checkpoint inhibitor, MK3475, which is a programmed cell death-1 (PD-1) inhibitor, has just started (NCT02178722). Two clinical trials of INCB024360 combined with cancer vaccines are recruiting patients: a phase II pilot study in combination with MELITAC 12.1, which is a multipeptide melanoma vaccine (NCT01961115); and a phase Ib/II study in combination with both CDX-140 [84], which is a vaccine for tumor antigen NY-ESO-1, and poly ICLC [84], which is a viral mimic composed of stabilized double-stranded RNA that acts as a ligand to Toll-like receptor 3 (NCT02166905).

Indoximod (NLG2101), which is D-1MT, is an orally administered IDO pathway inhibitor. An initial phase I study of indoximod as a single agent demonstrated good oral bioavailability and a favorable safety profile [85]. Based on these findings, a phase Ib trial of indoximod in combination with docetaxel (NCT01191216) was conducted in 27 patients; the results were reported at the ASCO annual meeting in 2013 [86]. The data showed that among 22 evaluable patients, $18 \%$ (4/22; 2 with breast cancer; 1, NSCLC; 1 , thymus cancer) exhibited a partial response, and $41 \%$ (9/22; 4 with breast cancer; 2 , NSCLC; 1, laryngeal cancer; 1 , esophageal cancer; 1 , ovarian cancer) had stable disease. Data indicated that the combination therapy was well tolerated with no increase in toxicities over those expected for the individual agents and no unexpected drug-drug interactions. Further, the pharmacokinetic profile of the combination therapy was similar to the profile of each drug as a single agent. Based on these results, a randomized phase II trial was initiated to evaluate the potential of indoximod in combination with docetaxel in patients with metastatic breast cancer (NCT01792050). Two more phase Ib/II trials of combined indoximod and chemotherapy are being conducted; indoximod plus albumin-bound paclitaxel (nab-paclitaxel) and gemcitabine in pancreatic cancer (NCT0277881) and indoximod plus temozolomide in brain tumors (NCT02052648).

Indoximod is also being tested in combination with other immunotherapy, such as a cancer vaccine or immune checkpoint inhibitor. First, a phase Ib study of combined indoximod and DC cancer vaccine (AD.p53DC) was presented at the ASCO annual meeting in 2013 [87]. The combination was well tolerated with no dose-limiting toxicities. The safety profile was consistent with the known monotherapy safety profiles for each agent. Among 32 patients enrolled in the phase I study, treatment discontinuations were all due to disease progression, not due to toxicities. No objective responses to the study therapy were noted, but after subsequent post-vaccine chemotherapy, 6 of 10 breast cancer patients treated with carboplatin/gemcitabine or gemcitabine alone showed response. This finding supports the hypothesis that immunotherapy treatments may sensitize patients to subsequent lines of therapy. Currently, a phase II trial is looking at the response to indoximod plus AD.p53DC vaccine followed by salvage carboplatin/gemcitabine in previously treated breast cancer patients (NCT01042535).

A second combination of indoximod with immunotherapy-a randomized, double-blind, phase II study of indoximod or placebo following infusion of sipuleucel$\mathrm{T}$-is being conducted in men with asymptomatic or minimally symptomatic metastatic castration-resistant prostate cancer (NCT01560923). Finally, another phase Ib/II study of combined indoximod and ipilimumab is recruiting patients with melanoma (NCT02073123).

Data from a phase I trial of an IDO peptide vaccine in 15 HLA-A2-positive patients with stage III-IV NSCLC were recently published [78]. The vaccine was well tolerated, with no severe toxicity. One patient developed a partial response, and 6 patients $(40 \%)$ demonstrated stable disease for $\geq 8.5$ months [78]. Meanwhile, a phase Ib study of combined IDO peptide vaccine and temozolomide is under way (NCT01543464).

NLG919 is the most recently developed orally bioavailable IDO1 inhibitor [79]. It was developed by the same company that developed the IDO pathway inhibitor indoximod. In preclinical setting, NLG919 was found to be as effective as indoximod in enhancing the survival in mice with glioblastoma and was synergistic when combined with standard chemo-radiation therapy [75]. Based on this preclinical finding, a phase I dose-escalating study is recruiting patients with advanced solid tumors (NCT02048709).

\section{Future directions}

Blockade of immune-inhibitory pathways, e.g., through inhibition of the IDO pathway, is emerging as an important modality for the treatment of cancer. However, single-agent treatments have partial anti-tumor activity 
in preclinical models and in cancer patients, as described above. The tumor microenvironment shows evidence of multiple immune-inhibitory mechanisms present concurrently such as PD-1/programmed cell death-ligand 1 (PD-L1) or cytotoxic T-lymphocyte-associated protein 4 (CTLA4) signaling as well as IDO signaling (Fig. 2) [82]. Remarkable clinical activity with rapid and deep tumor reduction has been reported in $65 \%$ of 53 patients with advanced melanoma treated with a combination of nivolumab, which is different from the published data on monotherapy PD-1 inhibitor, and ipilimumab, CTLA4 inhibitor [88]. We suggest that combination immunotherapies such as IDO inhibitors with other immune checkpoint inhibitors or cancer vaccines may be required for optimal therapeutic effect. In fact, combining IDO inhibitors with anti PD1/PD-L1 and anti-CTLA has increased the number of intratumoral $\mathrm{CD}^{+} \mathrm{T}$ cells, resulting in restored interleukin (IL) 2 production [82, 89]. From the standpoint of immunology, immunotherapy is considered a 'doubleedged sword' in that it can trigger two opposite immunologic processes. For example, among cytokines, which are major immune players, IL6 and IL10 are known to cause immune suppression or tolerance, leading to tumor progression, whereas IL2, IL12, IL23, interferon (INF)-alpha and INF-gamma are crucial in immune activation, leading to tumor suppression [90]. We may be able to better modulate the immune system by targeting both aspects: combining IDO inhibitors to block immune tolerance with immune activators such as IL2 or vaccines.

IDO inhibitors might work as single agents if IDO expression in the tumor microenvironment is the ratelimiting step and there are no other barriers to antitumor immune response, such as inadequate priming of the immune system, i.e., a lack of $\mathrm{CD} 8^{+} \mathrm{T}$ cells at the tumor site. Therefore, selection of biomarkers suggesting more benefit with IDO inhibitors, including expression of IDO and $\mathrm{CD}^{+} \mathrm{T}$ cells at the tumor site, should be investigated.

Another strategy to induce tumor sites to be more inflamed, or more primed for tumor immunity, is to administer targeted therapies, such as tyrosine kinase inhibitors. Frederick et al. reported that melanoma antigen expression and $\mathrm{CD}^{+} \mathrm{T}$ cells at the tumor sites were enhanced at the time of progression in patients with advanced melanoma treated with a BRAF inhibitor [91]. Interestingly, PD-L1 expression was also increased with BRAF inhibitor therapy, providing support for potential synergy of BRAF-targeted therapy and an immune checkpoint inhibitor. Further, as mentioned before, inhibition of IDO was demonstrated to contribute to the antitumor effects of imatinib in a preclinical model of gastrointestinal stromal tumor by suppressing Tregs and

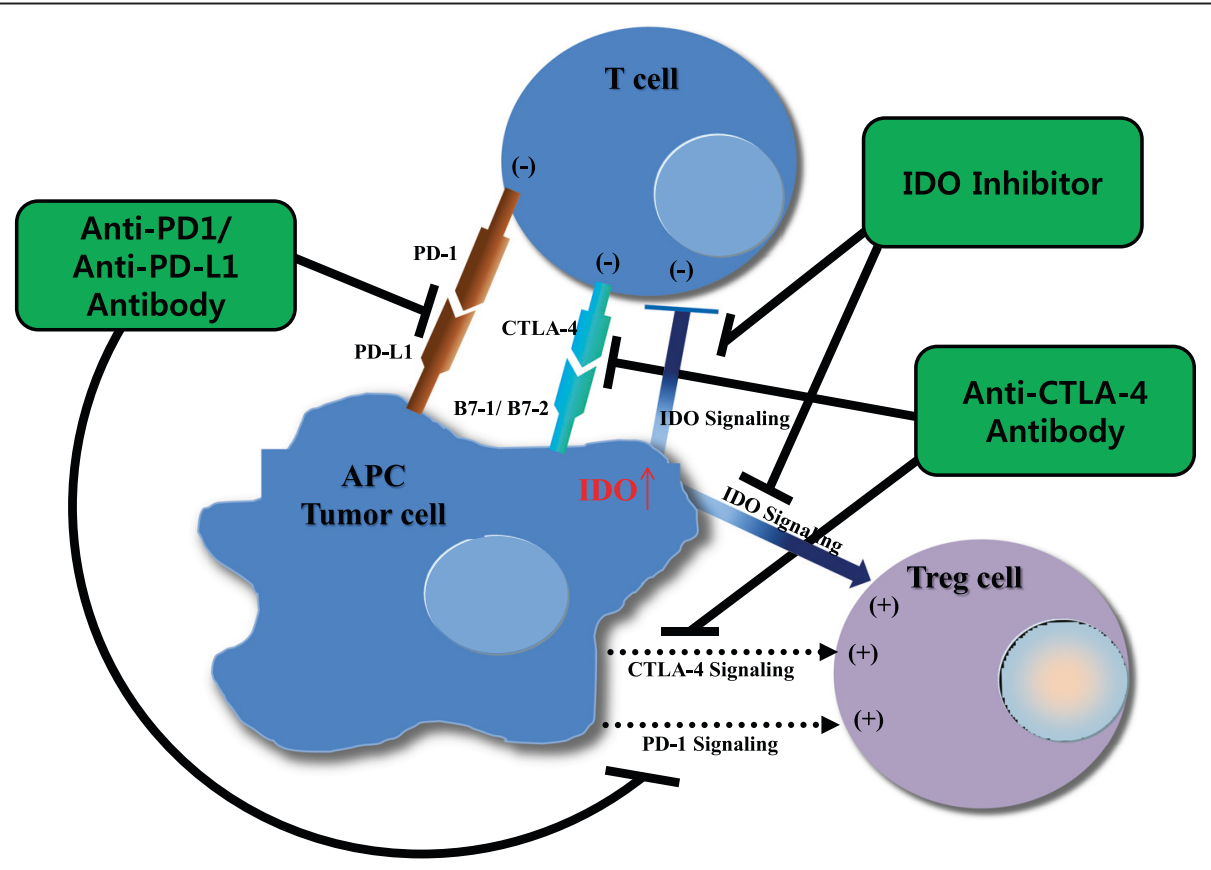

Fig. 2 Potential mechanism of combinatory synergism between three methods of immune checkpoint blockade. PD-1 or CTLA4 signaling inhibits proliferation of effector T cells. In addition, some studies have suggested that Tregs are also partly activated by PD-1 or CTLA4 signaling [100]. IDO signaling induces tumor immune tolerance by both suppressing effector T cells and activating Tregs. Therefore, if PD-1 or CTLA-4 signaling blockade is combined with an IDO inhibitor, effector T cells may be further activated and Tregs may be further suppressed. The result could be more effective reversal of immune tolerance and enhanced anti-tumor immune response. APC, antigen-presenting cell; PD-1, programmed cell death-1; PD-L1, programmed cell death-ligand 1; CTLA4, cytotoxic T-lymphocyte-associated protein 4; B7-1/2, peripheral membrane proteins found on activated APCS 
augmenting $\mathrm{CD}^{+} \mathrm{T}$ cells, which were partially induced by imatinib therapy [68]. A strategy of combining IDO inhibitors and various targeted therapies is strongly suggested for potential synergy.

As described above, in the phase I dose-escalating trials, IDO inhibitor monotherapy was generally very well tolerated. In addition to the excellent safety profile of IDO monotherapy, preclinical data that IDO inhibition helps overcome chemoresistance have prompted investigators to study combinations of an IDO inhibitor with other chemotherapeutic agents. As noted above, the combination of indoximod and docetaxel had a promising response rate in a preliminary report of the phase I trial [86].

\section{Conclusion}

Preclinical and preliminary clinical data demonstrates evidence to anti-tumor activity of IDO inhibitors. However, IDO inhibitors in combination with immunotherapies, targeted agents, and chemotherapy merit thorough investigation in cancer, and more clinical trials with immune monitoring are warranted.

\begin{abstract}
Abbreviations
1-MT: 1-methyl-DL-tryptophan; ASCO: American Society of Clinical Oncology; AHR: Aryl hydrocarbon receptor; BRAF: B-Raf proto-oncogene, serine/threonine kinase; CD: Cluster of differentiation; CTLA4: Cytotoxic T-lymphocyte-associated protein 4; DC: Dendritic cells; FOXP3 ${ }^{+}$: Forkhead box P3-positive; GCN2: General control nondepressible-2; GLK1: glucokinase; IDO: Indoleamine 2,3-dioxygenase; INF: Interferon; IL: Interleukin; mTORC1: mechanistic target of rapamycin complex 1; MLR: Mixed lymphocyte reaction; NSCLC: Non-small cell lung cancer; PD-1: Programmed cell death-1; PD-L1: Programmed cell death-ligand 1; PKC- $\theta$ : Protein kinase C theta; tRNA: Transfer ribonucleic acid; Tregs: $T$ regulatory cells; TDO: Tryptophan-2,3-dioxygenase; TDLN: Tumor draining lymph node.
\end{abstract}

\section{Competing interests}

The authors declare that they have no competing interests.

\section{Authors' contributions}

YWM drafted the manuscript. JH was involved in the writing and the critical review of the content of the manuscript. PH participated in the critical review of the content of the manuscript. AN was involved in the writing and critical review of the content of the manuscript. All authors read and provided final approval of the version to be published.

\section{Acknowledgements}

Authors would like to thank Sunita Patterson and Bettzy Stephen who helped with scientific editing of this manuscript.

\section{Author details}

${ }^{1}$ Medical Oncology, Department of Internal Medicine, CHA Bundang Medical Center, CHA University, Seongnam-si, Gyeonggi-do 463-712, South Korea. ${ }^{2}$ Department of Investigational Cancer Therapeutics, The University of Texas MD Anderson Cancer Center, 1515 Holcombe Blvd, Box 455, Houston, TX 77030, USA. ${ }^{3}$ Section of Immunology, Allergy \& Rheumatology, Baylor College of Medicine, Texas Children's Hospital, Houston, TX 77030, USA. ${ }^{4}$ Department of Melanoma Medical Oncology, The University of Texas MD Anderson Cancer Center, Houston, TX 77030, USA.

Received: 9 July 2015 Accepted: 13 October 2015 Published online: 15 December 2015

\section{References}

1. Lennerz V, Fatho M, Gentilini C, Frye RA, Lifke A, Ferel D, et al. The response of autologous $T$ cells to a human melanoma is dominated by mutated neoantigens. Proc Natl Acad Sci U S A. 2005;102(44):16013-8. doi:10.1073/ pnas.0500090102.

2. Ercolini AM, Ladle BH, Manning EA, Pfannenstiel LW, Armstrong TD, Machiels JP, et al. Recruitment of latent pools of high-avidity CD8(+) T cells to the antitumor immune response. J Exp Med. 2005;201(10):1591-602. doi:10.1084/jem.20042167.

3. Boon $T$, van der Bruggen P. Human tumor antigens recognized by $T$ lymphocytes. J Exp Med. 1996;183(3):725-9.

4. Zou W. Immunosuppressive networks in the tumour environment and their therapeutic relevance. Nat Rev Cancer. 2005;5(4):263-74. doi:10.1038/nrc1586.

5. Moffett JR, Namboodiri MA. Tryptophan and the immune response. Immunol Cell Biol. 2003;81(4):247-65. doi:10.1046/j.1440-1711.2003.t01-1-01177.x.

6. Yoshida R, Urade Y, Tokuda M, Hayaishi O. Induction of indoleamine 2,3-dioxygenase in mouse lung during virus infection. Proc Natl Acad Sci U S A. 1979;76(8):4084-6.

7. Yoshida R, Hayaishi O. Induction of pulmonary indoleamine 2,3-dioxygenase by intraperitoneal injection of bacterial lipopolysaccharide. Proc Natl Acad Sci U S A. 1978;75(8):3998-4000.

8. Pfefferkorn ER. Interferon gamma blocks the growth of Toxoplasma gondii in human fibroblasts by inducing the host cells to degrade tryptophan. Proc Natl Acad Sci U S A. 1984;81(3):908-12.

9. Theate I, van Baren N, Pilotte L, Moulin P, Larrieu P, Renauld JC, et al. Extensive profiling of the expression of the indoleamine 2,3-dioxygenase 1 protein in normal and tumoral human tissues. Cancer Immunol Res. 2015;3(2):161-72. doi:10.1158/2326-6066.CIR-14-0137.

10. Munn DH, Zhou M, Attwood JT, Bondarev I, Conway SJ, Marshall B, et al. Prevention of allogeneic fetal rejection by tryptophan catabolism. Science. 1998;281(5380):1191-3.

11. Platten M, Ho PP, Youssef S, Fontoura P, Garren H, Hur EM, et al. Treatment of autoimmune neuroinflammation with a synthetic tryptophan metabolite. Science. 2005;310(5749):850-5. doi:10.1126/science.1117634.

12. Guillonneau C, Hill M, Hubert FX, Chiffoleau E, Herve C, Li XL, et al. CD40lg treatment results in allograft acceptance mediated by CD8CD45RC T cells, IFN-gamma, and indoleamine 2,3-dioxygenase. J Clin Invest. 2007;117(4):1096-106. doi:10.1172/jci28801.

13. Cook CH, Bickerstaff AA, Wang JJ, Nadasdy T, Della Pelle P, Colvin RB, et al. Spontaneous renal allograft acceptance associated with "regulatory" dendritic cells and IDO. J Immunol. 2008;180(5):3103-12.

14. Hainz $U$, Jurgens B, Wekerle $T$, Seidel MG, Heitger A. Indoleamine 2,3dioxygenase in hematopoietic stem cell transplantation. Curr Drug Metab. 2007;8(3):267-72

15. Jasperson LK, Bucher C, Panoskaltsis-Mortari A, Taylor PA, Mellor AL, Munn $\mathrm{DH}$, et al. Indoleamine 2,3-dioxygenase is a critical regulator of acute graft-versus-host disease lethality. Blood. 2008;111(6):3257-65. doi:10.1182/blood-2007-06-096081.

16. Platten $M$, Wick W, Van den Eynde BJ. Tryptophan catabolism in cancer: beyond IDO and tryptophan depletion. Cancer Res. 2012;72(21):5435-40. doi:10.1158/0008-5472.can-12-0569.

17. Munn DH, Mellor AL. [Rev] Indoleamine 2,3 dioxygenase and metabolic control of immune responses. Trends Immunol. 2013;34(3):137-43. doi:10.1016/j.it.2012.10.001.

18. Heitger A. [Rev] Regulation of expression and function of IDO in human dendritic cells. Curr Med Chem. 2011;18(15):2222-33. doi: 0929-8673/11 \$58.00+.00.

19. Huang $L$, Baban $B$, Johnson 3rd BA, Mellor AL. Dendritic cells, indoleamine 2,3 dioxygenase and acquired immune privilege. Int Rev Immunol. 2010;29(2):133-55. doi:10.3109/08830180903349669.

20. Rutella S, Bonanno G, De Cristofaro R. Targeting indoleamine 2,3dioxygenase (IDO) to counteract tumour-induced immune dysfunction: from biochemistry to clinical development. Endocr Metab Immune Disord Drug Targets. 2009;9(2):151-77.

21. Johnson TS, Munn DH. Host indoleamine 2,3-dioxygenase: contribution to systemic acquired tumor tolerance. Immunol Invest. 2012;41(6-7):765-97. doi:10.3109/08820139.2012.689405.

22. Chung DJ, Rossi M, Romano E, Ghith J, Yuan J, Munn DH, et al. Indoleamine 2,3-dioxygenase-expressing mature human monocyte-derived dendritic cells expand potent autologous regulatory T cells. Blood. 2009;114(3):555-63. doi:10.1182/blood-2008-11-191197.

23. Prendergast GC, Smith C, Thomas S, Mandik-Nayak L, Laury-Kleintop L, Metz $R$, et al. Indoleamine 2,3-dioxygenase pathways of pathogenic inflammation and immune escape in cancer. Cancer Immunol Immunother. 2014;63(7):721-35. doi:10.1007/s00262-014-1549-4. 
24. Beutelspacher SC, Tan PH, McClure MO, Larkin DF, Lechler Rl, George AJ. Expression of indoleamine 2,3-dioxygenase (IDO) by endothelial cells: implications for the control of alloresponses. Am J Transplant. 2006;6(6):1320-30. doi:10.1111/j.1600-6143.2006.01324.x.

25. Chen JY, Li CF, Kuo CC, Tsai KK, Hou MF, Hung WC. Cancer/stroma interplay via cyclooxygenase-2 and indoleamine 2,3-dioxygenase promotes breast cancer progression. Breast Cancer Res. 2014;16(4):410. doi:10.1186/s13058-014-0410-1.

26. Hwu P, Du MX, Lapointe R, Do M, Taylor MW, Young HA. Indoleamine 2,3dioxygenase production by human dendritic cells results in the inhibition of T cell proliferation. J Immunol. 2000;164(7):3596-9.

27. Uyttenhove C, Pilotte L, Theate I, Stroobant V, Colau D, Parmentier N, et al. Evidence for a tumoral immune resistance mechanism based on tryptophan degradation by indoleamine 2,3-dioxygenase. Nat Med. 2003;9(10):1269-74. doi:10.1038/nm934.

28. Munn DH, Sharma MD, Lee JR, Jhaver KG, Johnson TS, Keskin DB, et al. Potential regulatory function of human dendritic cells expressing indoleamine 2,3-dioxygenase. Science. 2002;297(5588):1867-70. doi:10.1126/science.1073514.

29. Munn DH, Sharma MD, Hou D, Baban B, Lee JR, Antonia SJ, et al. Expression of indoleamine 2,3-dioxygenase by plasmacytoid dendritic cells in tumor-draining lymph nodes. J Clin Invest. 2004;114(2):280-90. doi:10.1172/jci21583.

30. Braun D, Longman RS, Albert ML. A two-step induction of indoleamine 2,3 dioxygenase (IDO) activity during dendritic-cell maturation. Blood. 2005;106(7):2375-81. doi:10.1182/blood-2005-03-0979.

31. Munn DH. Indoleamine 2,3-dioxygenase, Tregs and cancer. Curr Med Chem. 2011;18(15):2240-6. doi: 0929-8673/11 \$58.00+.00.

32. Metz R, Rust S, DuHadaway JB, Mautino MR, Munn DH, Vahanian NN, et al. IDO inhibits a tryptophan sufficiency signal that stimulates mTOR A novel IDO effector pathway targeted by D-1-methyl-tryptophan. Oncoimmunology. 2012;1(9):1460-8. doi:10.4161/onci.21716.

33. Chuang HC, Lan JL, Chen DY, Yang CY, Chen YM, Li JP, et al. The kinase GLK controls autoimmunity and NF-kappaB signaling by activating the kinase PKC-theta in T cells. Nat Immunol. 2011;12(11):1113-8. doi:10.1038/ni.2121.

34. Harding HP, Novoa I, Zhang Y, Zeng H, Wek R, Schapira M, et al. Regulated translation initiation controls stress-induced gene expression in mammalian cells. Mol Cell. 2000;6(5):1099-108.

35. Sundrud MS, Koralov SB, Feuerer M, Calado DP, Kozhaya AE, Rhule-Smith A, et al. Halofuginone inhibits TH17 cell differentiation by activating the amino acid starvation response. Science. 2009;324(5932):1334-8. doi:10.1126/ science.1172638.

36. Munn DH, Sharma MD, Baban B, Harding HP, Zhang Y, Ron D, et al. GCN2 kinase in T cells mediates proliferative arrest and anergy induction in response to indoleamine 2,3-dioxygenase. Immunity. 2005;22(5):633-42. doi:10.1016/j.immuni.2005.03.013.

37. Sharma MD, Baban B, Chandler P, Hou DY, Singh N, Yagita $H$, et al Plasmacytoid dendritic cells from mouse tumor-draining lymph nodes directly activate mature Tregs via indoleamine 2,3-dioxygenase. J Clin Invest. 2007;117(9):2570-82. doi:10.1172/JCl31911.

38. Nguyen NT, Kimura A, Nakahama T, Chinen I, Masuda K, Nohara K, et al. Aryl hydrocarbon receptor negatively regulates dendritic cell immunogenicity via a kynurenine-dependent mechanism. Proc Natl Acad Sci U S A. 2010;107(46):19961-6. doi:10.1073/pnas.1014465107.

39. Opitz CA, Litzenburger UM, Sahm F, Ott M, Tritschler I, Trump S, et al. An endogenous tumour-promoting ligand of the human aryl hydrocarbon receptor. Nature. 2011;478(7368):197-203. doi:10.1038/nature10491.

40. Sharma MD, Hou DY, Liu Y, Koni PA, Metz R, Chandler P, et al. Indoleamine 2,3-dioxygenase controls conversion of Foxp3+ Tregs to TH17-like cells in tumor-draining lymph nodes. Blood. 2009;113(24):6102-11. doi:10.1182/ blood-2008-12-195354.

41. Sharma MD, Hou DY, Baban B, Koni PA, He Y, Chandler PR, et al. Reprogrammed foxp3(+) regulatory $T$ cells provide essential help to support cross-presentation and CD8(+) T cell priming in naive mice. Immunity. 2010;33(6):942-54. doi:10.1016/j.immuni.2010.11.022.

42. Munn DH. Blocking IDO activity to enhance anti-tumor immunity. Front Biosci. 2012;4:734-45

43. Munn DH, Mellor AL, Rossi M, Young JW. Dendritic cells have the option to express IDO-mediated suppression or not. Blood. 2005;105(6):2618. doi:10.1182/blood-2005-01-0122.

44. Fallarino F, Grohmann U, You S, McGrath BC, Cavener DR, Vacca C, et al. Tryptophan catabolism generates autoimmune-preventive regulatory $T$ cells. Transpl Immunol. 2006;17(1):58-60. doi:10.1016/j.trim.2006.09.017.
45. Chen W, Liang X, Peterson AJ, Munn DH, Blazar BR. The indoleamine 2,3dioxygenase pathway is essential for human plasmacytoid dendritic cell-induced adaptive T regulatory cell generation. J Immunol. 2008;181(8):5396-404.

46. Manches O, Munn D, Fallahi A, Lifson J, Chaperot L, Plumas J, et al. HIV-activated human plasmacytoid DCs induce Tregs through an indoleamine 2,3-dioxygenase-dependent mechanism. J Clin Invest. 2008;118(10):3431-9. doi:10.1172/jci34823.

47. Schroecksnadel K, Frick B, Winkler C, Fuchs D. Crucial role of interferongamma and stimulated macrophages in cardiovascular disease. Curr Vasc Pharmacol. 2006:4(3):205-13.

48. Brandacher G, Hoeller E, Fuchs D, Weiss HG. Chronic immune activation underlies morbid obesity: is IDO a key player? Curr Drug Metab. 2007:8(3):289-95.

49. Pertovaara M, Raitala A, Juonala M, Lehtimaki T, Huhtala H, Oja SS, et al. Indoleamine 2,3-dioxygenase enzyme activity correlates with risk factors for atherosclerosis: the Cardiovascular Risk in Young Finns Study. Clin Exp Immunol. 2007;148(1):106-11. doi:10.1111/j.1365-2249.2007.03325.x.

50. Quan J, Tan PH, MacDonald A, Friend PJ. Manipulation of indoleamine 2,3dioxygenase (IDO) for clinical transplantation: promises and challenges. Expert Opin Biol Ther. 2008;8(11):1705-19. doi:10.1517/14712598.8.11.1705.

51. Curti A, Trabanelli S, Salvestrini $V$, Baccarani M, Lemoli RM. The role of indoleamine 2,3-dioxygenase in the induction of immune tolerance: focus on hematology. Blood. 2009;113(11):2394-401. doi:10.1182/blood-2008-07144485 .

52. Myint AM, Schwarz MJ, Steinbusch HW, Leonard BE. Neuropsychiatric disorders related to interferon and interleukins treatment. Metab Brain Dis. 2009:24(1):55-68. doi:10.1007/s11011-008-9114-5.

53. Dolusic E, Larrieu P, Moineaux L, Stroobant V, Pilotte L, Colau D, et al. Tryptophan 2,3-dioxygenase (TDO) inhibitors. 3-(2-(pyridyl)ethenyl)indoles as potential anticancer immunomodulators. J Med Chem. 2011;54(15):5320-34. doi:10.1021/jm2006782.

54. Mellor AL, Keskin DB, Johnson T, Chandler P, Munn DH. Cells expressing indoleamine 2,3-dioxygenase inhibit T cell responses. J Immunol. 2002;168(8):3771-6.

55. Sun JX, Zhang WG, Chen YX, Zhao WH, Tian W, Yang Y, et al. Indoleamine 2,3-dioxygenase expression in cells of human acute monocyte leukemia $(M(5))$ and acute lymphocyte leukemia and therapeutic effect of its inhibitor 1-methyl tryptophan. Zhongguo shi yan xue ye xue za zhi / Zhongguo bing li sheng li xue hui = Journal of experimental hematology / Chinese Association of. Pathophysiology. 2007;15(3):478-82.

56. Curti A, Aluigi $M$, Pandolfi S, Ferri E, Isidori A, Salvestrini $V$, et al. Acute myeloid leukemia cells constitutively express the immunoregulatory enzyme indoleamine 2,3-dioxygenase. Leukemia. 2007;21(2):353-5. doi:10.1038/sj.leu.2404485.

57. Sadun RE, Sachsman SM, Chen X, Christenson KW, Morris WZ, Hu P, et al Immune signatures of murine and human cancers reveal unique mechanisms of tumor escape and new targets for cancer immunotherapy. Clin Cancer Res. 2007;13(13):4016-25. doi:10.1158/1078-0432.ccr-07-0016.

58. Schroecksnadel K, Winkler C, Fuith LC, Fuchs D. Tryptophan degradation in patients with gynecological cancer correlates with immune activation. Cancer Lett. 2005;223(2):323-9. doi:10.1016/j.canlet.2004.10.033.

59. Corm S, Berthon C, Imbenotte M, Biggio V, Lhermitte M, Dupont C, et al. Indoleamine 2,3-dioxygenase activity of acute myeloid leukemia cells can be measured from patients' sera by HPLC and is inducible by IFN-gamma. Leuk Res. 2009;33(3):490-4. doi:10.1016/j.leukres.2008.06.014

60. Brandacher G, Perathoner A, Ladurner R, Schneeberger S, Obrist P, Winkler $C$, et al. [IDO in tumor] Prognostic value of indoleamine 2,3-dioxygenase expression in colorectal cancer: effect on tumor-infiltrating T cells. Clin Cancer Res. 2006;12(4):1144-51. doi:10.1158/1078-0432.ccr-05-1966.

61. Huang A, Fuchs D, Widner B, Glover C, Henderson DC, Allen-Mersh TG. Serum tryptophan decrease correlates with immune activation and impaired quality of life in colorectal cancer. Br J Cancer. 2002;86(11):1691-6. doi:10.1038/sj.bjc.6600336.

62. Ino K, Yamamoto E, Shibata K, Kajiyama H, Yoshida N, Terauchi M, et al. Inverse correlation between tumoral indoleamine 2,3-dioxygenase expression and tumor-infiltrating lymphocytes in endometrial cancer: its association with disease progression and survival. Clin Cancer Res. 2008;14(8):2310-7. doi:10.1158/1078-0432.ccr-07-4144.

63. Ino K, Yoshida N, Kajiyama H, Shibata K, Yamamoto E, Kidokoro K, et al. Indoleamine 2,3-dioxygenase is a novel prognostic indicator for endometrial cancer. Br J Cancer. 2006;95(11):1555-61. doi:10.1038/sj.bjc.6603477. 
64. Astigiano S, Morandi B, Costa R, Mastracci L, D'Agostino A, Ratto GB, et al. Eosinophil granulocytes account for indoleamine 2,3-dioxygenase-mediated immune escape in human non-small cell lung cancer. Neoplasia. 2005;7(4):390-6.

65. Weinlich G, Murr C, Richardsen L, Winkler C, Fuchs D. Decreased serum tryptophan concentration predicts poor prognosis in malignant melanoma patients. Dermatology. 2007;214(1):8-14. doi:10.1159/000096906.

66. Takao M, Okamoto A, Nikaido T, Urashima M, Takakura S, Saito M, et al. [IDO in tumor] Increased synthesis of indoleamine-2,3-dioxygenase protein is positively associated with impaired survival in patients with serous-type, but not with other types of, ovarian cancer. Oncol Rep. 2007;17(6):1333-9.

67. Okamoto A, Nikaido T, Ochiai K, Takakura S, Saito M, Aoki Y, et al. Indoleamine 2,3-dioxygenase serves as a marker of poor prognosis in gene expression profiles of serous ovarian cancer cells. Clin Cancer Res. 2005;11(16):6030-9. doi:10.1158/1078-0432.ccr-04-2671.

68. Balachandran VP, Cavnar MJ, Zeng S, Bamboat ZM, Ocuin LM, Obaid H, et al. Imatinib potentiates antitumor T cell responses in gastrointestina stromal tumor through the inhibition of Ido. Nat Med. 2011;17(9):1094-100. doi:10.1038/nm.2438

69. Hou DY, Muller AJ, Sharma MD, DuHadaway J, Banerjee T, Johnson M, et al. Inhibition of indoleamine 2,3-dioxygenase in dendritic cells by stereoisomers of 1-methyl-tryptophan correlates with antitumor responses. Cancer Res. 2007;67(2):792-801. doi:10.1158/0008-5472.can-06-2925.

70. Ball HJ, Sanchez-Perez A, Weiser S, Austin CJ, Astelbauer F, Miu J, et al. Characterization of an indoleamine 2,3-dioxygenase-like protein found in humans and mice. Gene. 2007;396(1):203-13. doi:10.1016/j.gene.2007.04.010.

71. Metz R, Duhadaway JB, Kamasani U, Laury-Kleintop L, Muller AJ, Prendergast GC. Novel tryptophan catabolic enzyme IDO2 is the preferred biochemical target of the antitumor indoleamine 2,3-dioxygenase inhibitory compound D-1-methyl-tryptophan. Cancer Res. 2007;67(15):7082-7. doi:10.1158/0008-5472.can-07-1872.

72. Lob S, Konigsrainer A, Schafer R, Rammensee HG, Opelz G, Terness P. Levo- but not dextro-1-methyl tryptophan abrogates the IDO activity of human dendritic cells. Blood. 2008;111(4):2152-4. doi:10.1182/blood-2007-10-116111.

73. Lob S, Konigsrainer A, Zieker D, Brucher BL, Rammensee HG, Opelz G, et al. IDO1 and IDO2 are expressed in human tumors: levo- but not dextro-1-methyl tryptophan inhibits tryptophan catabolism. Cancer Immunol Immunother. 2009;58(1):153-7. doi:10.1007/s00262-008-0513-6.

74. Lob S, Konigsrainer A, Rammensee HG, Opelz G, Terness P. Inhibitors of indoleamine-2,3-dioxygenase for cancer therapy: can we see the wood for the trees? Nat Rev Cancer. 2009;9(6):445-52. doi:10.1038/nrc2639.

75. Li M, Bolduc AR, Hoda MN, Gamble DN, Dolisca SB, Bolduc AK, et al. The indoleamine 2,3-dioxygenase pathway controls complement-dependent enhancement of chemo-radiation therapy against murine glioblastoma. J Immunother Cancer. 2014;2:21. doi:10.1186/2051-1426-2-21.

76. Liu X, Shin N, Koblish HK, Yang G, Wang Q, Wang K, et al. [INCB preclin] Selective inhibition of IDO1 effectively regulates mediators of antitumor immunity. Blood. 2010;115(17):3520-30. doi:10.1182/blood-2009-09-246124.

77. Koblish HK, Hansbury MJ, Bowman KJ, Yang G, Neilan CL, Haley PJ, et al. [INCB preclin] Hydroxyamidine inhibitors of indoleamine-2,3-dioxygenase potently suppress systemic tryptophan catabolism and the growth of IDO-expressing tumors. Mol Cancer Ther. 2010;9(2):489-98. doi:10.1158/ 1535-7163.mct-09-0628.

78. Iversen TZ, Engell-Noerregaard L, Ellebaek E, Andersen R, Larsen SK, Bjoern $J$ et al. [IDO pep vac] Long-lasting disease stabilization in the absence of toxicity in metastatic lung cancer patients vaccinated with an epitope derived from indoleamine 2,3 dioxygenase. Clin Cancer Res. 2013. doi:10.1158/1078-0432.ccr-13-1560.

79. Mautino MR, Jaipuri FA, Waldo J, Kumar S, Adams J, Allen CV et al. NLG919, a novel indoleamine-2,3-dioxygenase (IDO)-pathway inhibitor drug candidate for cancer therapy. Cancer Res. 2013;73(8 suppl;abstr 491). doi:10.1158/1538-7445.AM2013-491.

80. Beatty GL, O'Dwyer PJ, Clark J, Shi JG, Newton RC, Schaub R, et al. [INCB phl] Phase I study of the safety, pharmacokinetics (PK), and pharmacodynamics (PD) of the oral inhibitor of indoleamine 2,3dioxygenase (IDO1) INCB024360 in patients (pts) with advanced malignancies. J Clin Oncol. 2013;31(15_suppl):3025.

81. Castro MG, Baker GJ, Lowenstein PR. [Rev] Blocking immunosuppressive checkpoints for glioma therapy: the more the merrier! Clin Cancer Res. 2014:20(20):5147-9. doi:10.1158/1078-0432.ccr-14-0820.

82. Spranger S, Koblish HK, Horton B, Scherle PA, Newton R, Gajewski TF. Mechanism of tumor rejection with doublets of CTLA-4, PD-1/PD-L1, or IDO blockade involves restored IL-2 production and proliferation of CD8(+) T cells directly within the tumor microenvironment. J Immunother Cancer. 2014;2:3. doi:10.1186/2051-1426-2-3.

83. Gibney GT, Hamid O, Gangadhar TC, Lutzky J, Olszanski AJ, Gajewski T, et al. Preliminary results from a phase $1 / 2$ study of INCB024360 combined with ipilimumab (ipi) in patients (pts) with melanoma. J Clin Oncol. 2014;32(5_suppl):3010.

84. Dhodapkar MV, Sznol M, Zhao B, Wang D, Carvajal RD, Keohan ML, et al. Induction of antigen-specific immunity with a vaccine targeting NY-ESO-1 to the dendritic cell receptor DEC-205. Sci Transl Med. 2014;6(232):232ra51. doi:10.1126/scitranslmed.3008068.

85. Soliman HH, Neuger A, Noyes D, Vahanian NN, Link CJ, Munn D, et al. [Indocimod phl] A phase I study of 1-methyl-D-tryptophan in patients with advanced malignancies. J Clin Oncol. 2012;30(suppl):2501.

86. Jackson E, Dees EC, Kauh JS, Harvey RD, Neuger A, Lush R, et al. [indoximod + doce phl] A phase I study of indoximod in combination with docetaxel in metastatic solid tumors. J Clin Oncol. 2013;30(15_suppl):3026.

87. Soliman HH, Minton SE, Han HS, Ismail-Khan R, Mahipal A, Janssen W, et al. [Indoximod + p53vac phl] A phase I study of ad.p53 DC vaccine in combination with indoximod in metastatic solid tumors. J Clin Oncol. 2013;31(15 suppl):3069

88. Wolchok JD, Kluger H, Callahan MK, Postow MA, Rizvi NA, Lesokhin AM, et al. Nivolumab plus ipilimumab in advanced melanoma. N Engl J Med. 2013;369(2):122-33. doi:10.1056/NEJMoa1302369.

89. Holmgaard RB, Zamarin D, Munn DH, Wolchok JD, Allison JP. Indoleamine 2,3-dioxygenase is a critical resistance mechanism in antitumor $\mathrm{T}$ cell immunotherapy targeting CTLA-4. J Exp Med. 2013;210(7):1389-402. doi:10.1084/jem.20130066.

90. Vesely MD, Kershaw MH, Schreiber RD, Smyth MJ. Natural innate and adaptive immunity to cancer. Annu Rev Immunol. 2011;29:235-71. doi:10.1146/annurev-immunol-031210-101324.

91. Frederick DT, Piris A, Cogdill AP, Cooper ZA, Lezcano C, Ferrone CR, et al. BRAF Inhibition is associated with enhanced melanoma antigen expression and a more favorable tumor microenvironment in patients with metastatic melanoma. Clin Cancer Res. 2013;19(5):1225-31. doi:10.1158/1078-0432.Ccr-12-1630.

92. Okamoto T, Tone S, Kanouchi H, Miyawaki C, Ono S, Minatogawa Y. Transcriptional regulation of indoleamine 2,3-dioxygenase (IDO) by tryptophan and its analogue : Down-regulation of the indoleamine 2,3dioxygenase (IDO) transcription by tryptophan and its analogue. Cytotechnology. 2007;54(2):107-13. doi:10.1007/s10616-007-9081-4.

93. Eguchi $N$, Watanabe $Y$, Kawanishi $K$, Hashimoto $Y$, Hayaishi O. Inhibition of indoleamine 2,3-dioxygenase and tryptophan 2,3-dioxygenase by betacarboline and indole derivatives. Arch Biochem Biophys. 1984;232(2):602-9.

94. Kumar S, Malachowski WP, DuHadaway JB, LaLonde JM, Carroll PJ, Jaller D, et al. Indoleamine 2,3-Dioxygenase Is the Anticancer Target for a Novel Series of Potent Naphthoquinone-Based Inhibitors (vol 51, pg 1712, 2008). J Med Chem. 2008;51(22):7325. doi:10.1021/Jm8013053.

95. Gaspari P, Banerjee T, Malachowski WP, Muller AJ, Prendergast GC, DuHadaway J, et al. Structure-activity study of Brassinin derivatives as indoleamine 2,3-dioxygenase inhibitors. J Med Chem. 2006;49(2):684-92. doi:10.1021/Jm0508888.

96. Banerjee T, DuHadaway JB, Gaspari P, Sutanto-Ward E, Munn DH, Mellor AL, et al. A key in vivo antitumor mechanism of action of natural product-based brassinins is inhibition of indoleamine 2,3-dioxygenase. Oncogene. 2008:27(20):2851-7. doi:10.1038/sj.onc.1210939.

97. Sono M, Cady SG. Enzyme kinetic and spectroscopic studies of inhibitor and effector interactions with indoleamine 2,3-dioxygenase .1. Norharman and 4-Phenylimidazole binding to the enzyme as inhibitors and heme ligands. Biochemistry (Mosc). 1989;28(13):5392-9. doi:10.1021/Bi00439a012.

98. Brastianos HC, Vottero E, Patrick BO, Van Soest R, Matainaho T, Mauk AG, et al. Exiguamine A, an indoleamine-2,3-dioxygenase (IDO) inhibitor isolated from the marine sponge Neopetrosia exigua. J Am Chem Soc. 2006;128(50):16046-7. doi:10.1021/ja067211+.

99. Vottero E, Balgi A, Woods K, Tugendreich S, Melese T, Andersen RJ, et al. Inhibitors of human indoleamine 2,3-dioxygenase identified with a targetbased screen in yeast. Biotechnol J. 2006;1(3):282-8. doi:10.1002/ biot.200600001.

100. Nishikawa H, Sakaguchi S. Regulatory T cells in cancer immunotherapy. Curr Opin Immunol. 2014;27:1-7. doi:10.1016/j.coi.2013.12.005. 